\title{
A New Uncertainty Evaluation Method and Its Application in Evaluating Software Quality
}

\author{
Jiqiang Chen, ${ }^{1}$ Litao Ma, ${ }^{1}$ Chao Wang, ${ }^{2}$ Hong Zhang, ${ }^{1}$ and Jie Wan ${ }^{3}$ \\ ${ }^{1}$ School of Science, Hebei University of Engineering, Handan 056038, China \\ ${ }^{2}$ School of Economics and Management, Hebei University of Engineering, Handan 056038, China \\ ${ }^{3}$ School of Energy Science and Engineering, Harbin Institute of Technology, Harbin 150001, China \\ Correspondence should be addressed to Litao Ma; ltma1821@163.com
}

Received 6 February 2014; Accepted 28 May 2014; Published 2 July 2014

Academic Editor: Chao Yan

Copyright (C) 2014 Jiqiang Chen et al. This is an open access article distributed under the Creative Commons Attribution License, which permits unrestricted use, distribution, and reproduction in any medium, provided the original work is properly cited.

\begin{abstract}
Uncertainty theory is a branch of axiomatic mathematics dealing with experts' belief degree. Considering the uncertainty with experts' belief degree in the evaluation system and the different roles which different indices play in evaluating the overall goal with a hierarchical structure, a new comprehensive evaluation method is constructed based on uncertainty theory. First, index scores and weights of indices are described by uncertain variables and evaluation grades are described by uncertain sets. Second, weights of indices with respect to the overall goal are introduced. Third, a new uncertainty comprehensive evaluation method is constructed and proved to be a generalization of the weighted average method. Finally, an application is developed in evaluating software quality, which shows the effectiveness of the new method.
\end{abstract}

\section{Introduction}

Due to human language and technology difficulties, it is difficult to provide an effect and objective evaluation for a system. Therefore, many scholars attempt to establish new mathematical methods to make evaluation results consistent with actual situations. Accordingly, Saaty [1] proposed the analytic hierarchy process (AHP), a method to address multicriteria decision analysis with quantitative and qualitative information. In AHP, human judgments are represented as exact numbers. However, some decision-makers may be reluctant or unable to assign exact numbers to comparison judgments because some evaluation criteria are subjective and qualitative. Therefore, Wu and Tsai [2] used both AHP and decisionmaking trial and evaluation laboratory methods to evaluate the criteria in autospare parts industry in Taiwan. Kumar et al. [3] presented a new general procedure to construct the membership and nonmembership functions of the fuzzy reliability using time-dependent intuitionistic fuzzy set. By using the finite Markov chain imbedding approach, Zhao and Cui [4] presented a unified formula with the product of matrices for evaluating the system state distribution for generalized multistate $k$-out-of- $n$ : $F$ systems. Chen et al. [5] provided an evaluation method for enterprisers making investment decisions under hybrid cloud environment using grey system theory. Lee et al. [6] proposed a systematic approach to evaluation of new service concepts by integrating the merit of group analytic hierarchy process in modeling multicriteria decision-making problems. Geng et al. [7] presented a new integrated design concept evaluation approach based on vague sets in order to provide a method for complicated multicriteria decision-making problem under uncertain environments. Aiming to evaluate the government and the monopolist about the consumer's taste, literature [8] was devoted to the characterization and quantitative representation of imprecise and vague uncertainties and measures of information produced by sources of the considered type. Kramosil [9] introduced the possibilistic variants of both the minimax (the worst case) and the Bayesian optimization principles and applied them in decision-making under uncertainty processed. Using finite-time control and backstepping control approaches, $\mathrm{Li}$ et al. [10] proposed a new robust adaptive synchronization scheme to make the synchronization errors of the systems with parameter 
uncertainties zero in a finite time. Lan et al. [11] presented a bilevel fuzzy principal-agent model for optimal nonlinear taxation problems with asymmetric information and so on.

The above methods address imprecise information, such as human language or experts' degree of belief using fuzzy set theory (see Zadeh [12]), vague set theory, and grey system theory. However, for the evaluation system, the observed data are often not adequate and we have no choice but to invite some domain experts to evaluate the belief degree that an index belongs to an evaluation grade. In this situation, many surveys show that this imprecise information behaves like neither fuzziness nor randomness (see Liu [13] and Liu [14]). And it was showed by Kahneman and Tversky [15] that human beings usually overweight unlikely events. This fact makes the personal belief degree have much larger variance than the frequency.

In this case, Liu [13] proposed uncertainty theory to deal with belief degree, and Liu [14] refined uncertainty theory. Nowadays, uncertainty theory has become a branch of axiomatic mathematics. The first fundamental concept in uncertainty theory is the uncertain measure, used to measure the degree of belief in an event. The second concept is the uncertain variable, used to represent quantities with imprecise information (e.g., the exact value of oil field reserve). The third concept is uncertainty distribution, which is used to describe uncertain variables. Uncertainty theory has been applied to many areas. Liu [16] established a theory and practice of uncertain programming, Liu [17] applied uncertainty theory to risk analysis and reliability analysis, Liu [18] studied hybrid logic and uncertain logic, Liu [19] proposed inference rule with applications to uncertain control, and Liu [20] studied uncertain process with applications to inference risk model. To explore the recent developments in uncertainty theory, readers may consult Liu [21].

Subsequently, Liu [22] described the weights of indices and the score values of indices with uncertain variables and proposed a comprehensive evaluation method based on uncertainty theory. However, in some certain kinds of assessment domains, we find that different bottom indices play different roles in the evaluation of the overall goal. For example, suppose that $S_{1}$ and $S_{2}$ are two students whose four features are shown in Table 1.

Therefore, the feature vectors of $S_{1}$ and $S_{2}$ are $V_{1}=$ $(1,20,180,80)$ and $V_{2}=(1,20,170,75)$. Because $S_{1}$ and $S_{2}$ are with the same "Gender" and "Age," we cannot identify them from the two features. In other words, "Gender" and "Age" do not take effect at all in the identification. Furthermore, because the "Body Height" of $S_{1}$ and $S_{2}$ is 180 and 170 , respectively, they can be identified by "Body Height." Of course, they can also be identified by "Body Weight." In a word, the four features play different roles in the identification of $S_{1}$ and $S_{2}$. For this reason, a weight for each bottom index with respect to the overall goal is introduced to show the different roles of different bottom indices. And without loss of generality, when different bottom indices play the same role in other assessment domains, weights of indices with respect to the overall goal are equal. Considering these reasons, a new evaluation method is proposed based on uncertainty theory.
TABLE 1: Four features of students $S_{1}$ and $S_{2}$.

\begin{tabular}{lcccc}
\hline Students & $\begin{array}{c}\text { Gender } \\
(\text { male }=1, \text { female }=0)\end{array}$ & $\begin{array}{c}\text { Age } \\
(\text { years })\end{array}$ & $\begin{array}{c}\text { Body height } \\
(\mathrm{cm})\end{array}$ & $\begin{array}{c}\text { Body weight } \\
(\mathrm{kg})\end{array}$ \\
\hline$S_{1}$ & 1 & 20 & 180 & 80 \\
$S_{2}$ & 1 & 20 & 170 & 75 \\
\hline
\end{tabular}

The structure of this paper is as follows. Section 2 provides some relevant concepts about uncertainty theory. Section 3 establishes a new uncertainty evaluation method based on uncertain sets and uncertain variables. Section 4 gives an application of the proposed method in evaluating software quality, and the conclusions are presented in Section 5 .

\section{Preliminaries}

In this section, we provide some useful definitions of uncertainty theory.

Let $\Gamma$ be a nonempty set, let $\mathscr{L}$ be a $\sigma$-algebra on $\Gamma$, and let $R$ be a set of real numbers. Each element $\Lambda \in \mathscr{L}$ is called an event. The uncertain measure $\mathscr{M}$, defined on $\mathscr{L}$, was proposed by Liu [13] as follows.

Definition 1 (see Liu [13]). The set function $\mathscr{M}$ is called an uncertain measure if it satisfies the following.

Axiom 1 (normality axiom). Consider $\mathscr{M}\{\Gamma\}=1$.

Axiom 2 (duality axiom). Consider $\mathscr{M}\{\Lambda\}+\mathscr{M}\left\{\Lambda^{c}\right\}=1$ for any event $\Lambda$.

Axiom 3 (subadditivity axiom). For every countable sequence of events $\left\{\Lambda_{i}\right\}$, we have

$$
\mathscr{M}\left\{\bigcup_{i=1}^{\infty} \Lambda_{i}\right\} \leq \sum_{i=1}^{\infty} \mathscr{M}\left\{\Lambda_{i}\right\} .
$$

The triplet $(\Gamma, \mathscr{L}, \mathscr{M})$ is called an uncertainty space. Let $\left(\Gamma_{k}, \mathscr{L}_{k}, \mathscr{M}_{k}\right)$ be uncertainty spaces for $k=1,2, \ldots$. Write

$$
\Gamma=\Gamma_{1} \times \Gamma_{2}, \ldots, \quad \mathscr{L}=\mathscr{L}_{1} \times \mathscr{L}_{2} \times \cdots .
$$

Then the product uncertain measure $\mathscr{M}$ on the product $\sigma$ algebra $\mathscr{L}$ is defined by the following product axiom [23].

Axiom 4 (product axiom). Let $\left(\Gamma_{k}, \mathscr{L}_{k}, \mathscr{M}_{k}\right)$ be uncertainty spaces for $k=1,2, \ldots$. The product uncertain measure $\mathscr{M}$ is an uncertain measure satisfying

$$
\mathscr{M}\left\{\prod_{k=1}^{\infty} \Lambda_{k}\right\}=\bigwedge_{k=1}^{\infty} \mathscr{M}\left\{\Lambda_{k}\right\},
$$

where $\Lambda_{k}$ are arbitrarily chosen events from $\mathscr{L}_{k}$ for $k=$ $1,2, \ldots$, respectively.

Remark 2. Uncertain measure is interpreted as the personal belief degree (not frequency) of an uncertain event that may occur. It depends on the personal knowledge concerning the event. The uncertain measure will change if the state of knowledge changes. 


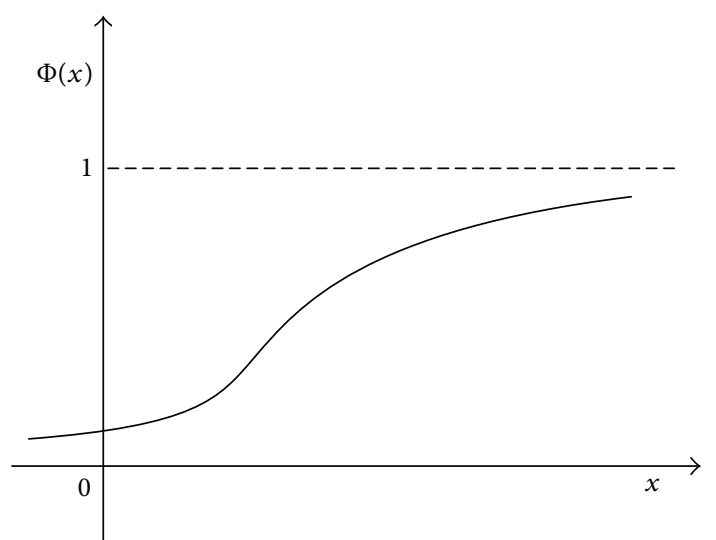

FIgURE 1: An uncertainty distribution.

Definition 3 (see Liu [13]). An uncertain variable is a measurable function $\xi$ from an uncertainty space $(\Gamma, \mathscr{L}, \mathscr{M})$ to the set of real numbers. That is, for any Borel set $B$ of real numbers, the set

$$
\{\xi \in B\}=\{\gamma \in \Gamma \mid \xi(\gamma) \in B\}
$$

is an event.

Definition 4 (see Liu [14]). Let $f$ be a real-valued measurable function, and let $\xi_{1}, \xi_{2}, \ldots, \xi_{n}$ be uncertain variables on $(\Gamma, \mathscr{L}, \mathscr{M})$. Then $\xi=f\left(\xi_{1}, \xi_{2}, \ldots, \xi_{n}\right)$ is an uncertain variable defined by

$$
\xi(\gamma)=f\left(\xi_{1}(\gamma), \xi_{2}(\gamma), \ldots, \xi_{n}(\gamma)\right), \quad \forall \gamma \in \Gamma .
$$

Definition 5 (see Liu [14]). The uncertainty distribution $\Phi$ of an uncertain variable $\xi$ is defined by

$$
\Phi(x)=\mathscr{M}\{\xi \leq x\}
$$

for any real number $x$ (see Figure 1).

Definition 6 (see Liu [14]). An uncertain variable $\xi$ is called zigzag if it has a zigzag uncertainty distribution

$$
\Phi(x)= \begin{cases}0, & \text { if } x \leq a \\ \frac{(x-a)}{2(b-a)}, & \text { if } a \leq x \leq b \\ \frac{(x+c-2 b)}{2(c-b)}, & \text { if } b \leq x \leq c \\ 1, & \text { if } x \geq c\end{cases}
$$

denoted by $Z(a, b, c)$, where $a, b, c$ are real numbers with $a<$ $b<c$.

Definition 7 (see Liu [23]). The uncertain variables $\xi_{1}, \xi_{2}, \ldots$, $\xi_{n}$ are said to be independent if

$$
\mathscr{M}\left\{\bigcap_{i=1}^{n}\left(\xi_{i} \in B_{i}\right)\right\}=\bigwedge_{i=1}^{n} \mathscr{M}\left\{\xi_{i} \in B_{i}\right\}
$$

for any Borel sets $B_{1}, B_{2}, \ldots, B_{n}$ of real numbers.
Theorem 8 (see Liu [21]). Assume that $\xi_{1}$ and $\xi_{2}$ are independent zigzag uncertain variables $Z\left(a_{1}, b_{1}, c_{1}\right)$ and $Z\left(a_{2}, b_{2}, c_{2}\right)$, respectively. Then the sum $\xi_{1}+\xi_{2}$ is also a zigzag uncertain variable $Z\left(a_{1}+a_{2}, b_{1}+b_{2}, c_{1}+c_{2}\right)$; that is,

$$
Z\left(a_{1}, b_{1}, c_{1}\right)+Z\left(a_{2}, b_{2}, c_{2}\right)=Z\left(a_{1}+a_{2}, b_{1}+b_{2}, c_{1}+c_{2}\right) \text {. }
$$

The product of a zigzag uncertain variable $Z(a, b, c)$ and $a$ scalar number $k>0$ is also a zigzag uncertain variable $Z(k a, k b, k c)$; that is,

$$
k Z(a, b, c)=Z(k a, k b, k c) .
$$

Definition 9 (see Liu [13]). Let $\xi$ be an uncertain variable. Then the expected value of $\xi$ is defined by

$$
E[\xi]=\int_{0}^{+\infty} \mathscr{M}\{\xi \geq r\} d r-\int_{-\infty}^{0} \mathscr{M}\{\xi \leq r\} d r
$$

provided that at least one of the two integrals is finite.

Remark 10. Expected value is the average value of uncertain variable in the sense of uncertain measure and represents the size of uncertain variable.

Example 11 (see Liu [21]). The zigzag uncertain variable $\xi \sim$ $Z(a, b, c)$ has an expected value

$$
E[\xi]=\frac{a+2 b+c}{4} .
$$

Theorem 12 (see Liu [14]). Let $\xi$ and $\eta$ be independent uncertain variables with finite expected values. Then for any real numbers $a$ and $b$, one has

$$
E[a \xi+b \eta]=a E[\xi]+b E[\eta] .
$$

An uncertain set is a set-valued function on an uncertainty space that attempts to model "unsharp concepts," which are essentially sets but their boundaries are not sharply described (because of the ambiguity of human language), such as "young" and "tall." A formal definition is given as follows.

Definition 13 (see Liu [19]). An uncertain set is a measurable function $\xi$ from an uncertainty space $(\Gamma, \mathscr{L}, \mathscr{M})$ to a collection of sets of real numbers. For any Borel set $B$ of real numbers, that is, for any Borel set $B$ of real numbers, both of

$$
\begin{aligned}
& \{\xi \subset B\}=\{\gamma \in \Gamma \mid \xi(\gamma) \subset B\}, \\
& \{B \subset \xi\}=\{\gamma \in \Gamma \mid B \subset \xi(\gamma)\}
\end{aligned}
$$

are events.

Definition 14 (see Liu [24]). An uncertain set $\xi$ is said to have a membership function $\mu$ if for any Borel set $B$ of real numbers one has

$$
\begin{gathered}
\mathscr{M}\{B \subset \xi\}=\inf _{x \in B} \mu(x), \\
\mathscr{M}\{\xi \subset B\}=1-\sup _{x \in B^{c}} \mu(x) .
\end{gathered}
$$

The above equations will be called measure inversion formulas. 


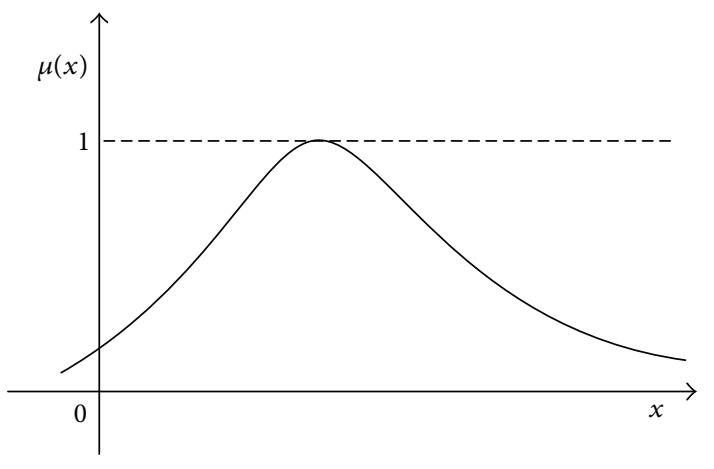

Figure 2: A membership function.

Remark 15. Let $\mathfrak{R}$ be a set of real numbers. When an uncertain set has a membership function $\mu$ on $\mathfrak{R}$, we immediately have

$$
\mu(x)=\mathscr{M}\{x \in \xi\}, \quad \forall x \in \mathfrak{R} .
$$

Liu [24] proved that a real-valued function $\mu$ is a membership function if and only if $0 \leq \mu(x) \leq 1$ (see Figure 2).

Example 16 (see Liu [21]). By a triangular uncertain set we mean the uncertain set fully determined by the triplet $(a, b, c)$ of crisp numbers with $a<b<c$, whose membership function is

$$
\mu(x)= \begin{cases}\frac{(x-a)}{(b-a)}, & \text { if } a \leq x \leq b, \\ \frac{(x-c)}{(b-c)}, & \text { if } b \leq x \leq c, \\ 0, & \text { otherwise. }\end{cases}
$$

Definition 17 (see Liu [24]). Let $\xi$ be an uncertain set with a membership function $\mu$. Then the set-valued function

$$
\mu^{-1}(\alpha)=\{x \in \mathfrak{R} \mid \mu(x) \geq \alpha\}, \quad \forall \alpha \in[0,1]
$$

is called the inverse membership function of $\xi$.

Definition 18 (see Liu [25]). The uncertain sets $\xi_{1}, \xi_{2}, \ldots, \xi_{n}$ are said to be independent if for any Borel sets $B_{1}, B_{2}, \ldots, B_{n}$ one has

$$
\begin{aligned}
& \mathscr{M}\left\{\bigcap_{i=1}^{n}\left(\xi_{i}^{*} \subset B_{i}\right)\right\}=\bigwedge_{i=1}^{n} \mathscr{M}\left\{\xi_{i}^{*} \subset B_{i}\right\} \\
& \mathscr{M}\left\{\bigcup_{i=1}^{n}\left(\xi_{i}^{*} \subset B_{i}\right)\right\}=\bigvee_{i=1}^{n} \mathscr{M}\left\{\xi_{i}^{*} \subset B_{i}\right\}
\end{aligned}
$$

where $\xi_{i}^{*}$ are arbitrarily chosen from $\left\{\xi_{i}, \xi_{i}^{c}\right\}, i=1,2, \ldots, n$, respectively.

Theorem 19 (see Liu [24]). Let $\xi$ and $\eta$ be independent uncertain sets with membership functions $\mu$ and $\nu$, respectively. Then their union $\xi \cup \eta$ has a membership function

$$
\lambda(x)=\mu(x) \vee v(x) \text {. }
$$

Theorem 20 (see Liu [24]). Let $\xi$ and $\eta$ be independent uncertain sets with membership functions $\mu$ and $\nu$, respectively. Then their intersection $\xi \cap \eta$ has a membership function

$$
\lambda(x)=\mu(x) \wedge \nu(x) .
$$

Theorem 21 (see Liu [24]). Let $\xi$ be an uncertain set with membership function $\mu$. Then its complement $\xi^{c}$ has a membership function

$$
\lambda(x)=1-\mu(x) .
$$

Theorem 22 (see Liu [24]). Let $\xi_{1}, \xi_{2}, \ldots, \xi_{n}$ be independent uncertain sets with inverse membership functions $\mu_{1}^{-1}, \mu_{2}^{-1}, \ldots, \mu_{n}^{-1}$, respectively. If the function $f$ is a measurable function, then

$$
\xi=f\left(\xi_{1}, \xi_{2}, \ldots, \xi_{n}\right)
$$

is an uncertain set with inverse membership function

$$
\lambda^{-1}(\alpha)=f\left(\mu_{1}^{-1}(\alpha), \mu_{2}^{-1}(\alpha), \ldots, \mu_{n}^{-1}(\alpha)\right) .
$$

Definition 23 (see Liu [19]). Let $\xi$ be a nonempty uncertain set. Then the expected value of $\xi$ is defined by

$$
E[\xi]=\int_{0}^{+\infty} \mathscr{M}\{\xi \succeq r\} d r-\int_{-\infty}^{0} \mathscr{M}\{\xi \preceq r\} d r
$$

provided that at least one of the two integrals is finite.

Please note that $\xi \succeq r$ represents " $\xi$ is imaginarily included in $[r,+\infty)$ " and $\xi \preceq r$ represents " $\xi$ is imaginarily included in $(-\infty, r]$."

Example 24 (see Liu [21]). The triangular uncertain set $\xi=$ $(a, b, c)$ has an expected value

$$
E[\xi]=\frac{a+2 b+c}{4} .
$$

\section{Uncertainty Evaluation Method}

When making a comprehensive evaluation, factors influencing the grade of the overall goal should be considered. The index system is often represented by a three-layer hierarchical structure with the overall goal, the second layer, and the bottom layer (namely, the factors influencing the overall goal's scaling). Experts always intend to show their own opinions and expectations of each evaluation index, so the evaluation results represent human uncertainty and belief degree. Therefore, the score value and weight of each evaluation index are represented by uncertain variables, and evaluation grades are represented by uncertain sets. Therefore, a new evaluation method based on uncertain variables and uncertain sets is proposed.

3.1. Establishment of Hierarchical Index Structure. Based on evaluation criteria, a hierarchical index structure is established by experts as in Figure 3. There are $l$ indices in the second level and $m_{i}$ indices immediately below index $B_{i}$ $(i=1,2, \ldots, l)$, where $m_{1}+m_{2}+\cdots+m_{l}=m$. Let the index (in the bottom level) set be $C=\left\{C_{11}, C_{12}, \ldots, C_{1 m_{1}}\right.$, $\left.C_{21}, C_{22}, \ldots, C_{2 m_{2}}, C_{l 1}, C_{l 2}, \ldots, C_{1 m_{l}}\right\}$. 


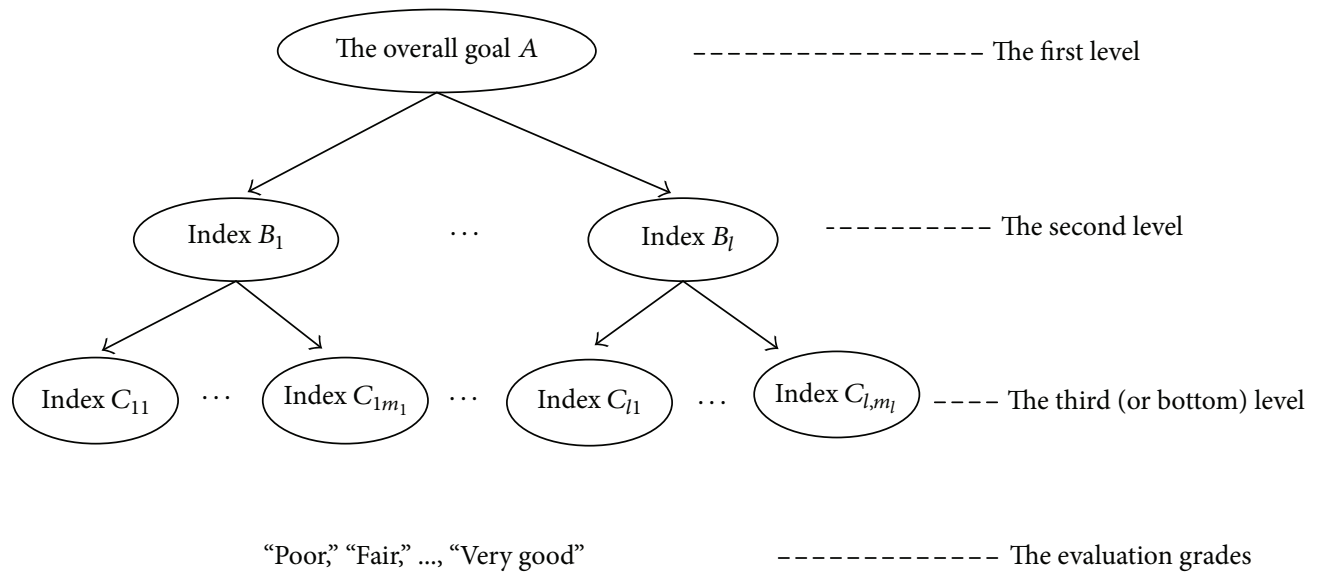

FIgURE 3: A simple hierarchical index structure.

3.2. Weights of Indices. Suppose that there are $r$ experts in the evaluation group and the $n$ degrees of importance are represented by uncertain variables $\eta_{1}, \eta_{2}, \ldots, \eta_{n}$. Each expert gives a degree of importance for each index. For index $C_{i j}$ $\left(i=1,2, \ldots, t ; j=1,2, \ldots, m_{i}\right), r_{1}$ experts select $\eta_{1}, r_{2}$ experts select $\eta_{2}, \ldots, r_{n}$ experts select $\eta_{n}$, where $r_{1}+r_{2}+\cdots+r_{n}=r$. Weight $w_{i j}$ of index $C_{i j}$ is represented by

$$
\begin{gathered}
w_{i j}=\frac{r_{1}}{r} \eta_{1}+\frac{r_{2}}{r} \eta_{2}+\cdots+\frac{r_{n}}{r} \eta_{n}, \\
i=1,2, \ldots, t ; j=1,2, \ldots, m_{i} .
\end{gathered}
$$

According to Definition 3, weight $w_{i j}$ is an uncertain variable and can be described by its uncertainty distribution $\Phi_{i j}$.

3.3. Grade Vectors of Bottom Indices. The score values from experts can be represented by uncertain variables. Let the $t$ evaluation grades (e.g., Poor, Fair,..., Excellent) be represented by uncertain sets $\xi_{1}, \xi_{2}, \ldots, \xi_{t}$, with membership functions $\mu_{1}, \mu_{2}, \ldots, \mu_{t}$, respectively. Let $x_{i j}$ be the index score of index $C_{i j}$. Then, the grade vector $s_{i j}$ of index $C_{i j}$ is represented by

$$
s_{i j}=\left(s_{i j 1}=\mu_{1}\left(x_{i j}\right), s_{i j 2}=\mu_{2}\left(x_{i j}\right), \ldots, s_{i j t}=\mu_{t}\left(x_{i j}\right)\right) \text {, }
$$

where $s_{i j k}(k=1,2, \ldots, t)$ is the membership degree to which index $C_{i j}$ belongs to grade $\xi_{k}$.

The next step is to construct a method to realize the transformation from the grade vector $s_{i j}$ of the bottom index $C_{i j}$ to the grade vector $s(A)$ of the overall goal $A$.

3.4. Transformation Method. The transformation method can be obtained with the following three steps.

Step 1. To determine the importance of index $C_{i j}$ in the grading of the overall goal, the weight of index $C_{i j}$ with respect to the overall goal $A$ is introduced beginning with the following formulas:

$$
\begin{gathered}
H_{i j}(A)=-\sum_{k=1}^{t} s_{i j k} \cdot \lg s_{i j k}, \\
V_{i j}(A)=1-\frac{1}{\lg t} H_{i j}(A), \\
\lambda_{i j}(A)=\frac{V_{i j}(A)}{\sum_{i=1}^{l} \sum_{j=1}^{m_{i}} V_{i j}(A)},
\end{gathered}
$$

where $\lambda_{i j}(A)$ is the weight of index $C_{i j}$ with respect to $A$. From the above formulas, we have

$$
0 \leq \lambda_{i j}(A) \leq 1, \quad \sum_{i=1}^{l} \sum_{j=1}^{m_{i}} \lambda_{i j}(A)=1 .
$$

Step 2. Because $\lambda_{i j}(A)$ shows the degree of the effective information offered by $C_{i j}$ in the evaluation of $A$, we calculate

$$
\lambda_{i j}(A) \cdot s_{i j k} \text {. }
$$

Step 3. By weight $w_{i j}\left(B_{i}\right)$ of index $C_{i j}$ with respect to $B_{i}$, we have

$$
\begin{gathered}
N_{k}\left(B_{i}\right)=\sum_{j=1}^{m_{i}} w_{i j}\left(B_{i}\right) \cdot \lambda_{i j}(A) \cdot s_{i j k}, \\
s_{k}\left(B_{i}\right)=\frac{N_{k}\left(B_{i}\right)}{\sum_{k=1}^{t} N_{k}\left(B_{i}\right)} .
\end{gathered}
$$

From the above formulas, we have

$$
0 \leq s_{k}\left(B_{i}\right) \leq 1, \quad \sum_{k=1}^{t} s_{k}\left(B_{i}\right)=1 .
$$

Thus, we obtain the grade vector $s\left(B_{i}\right)=\left(s_{1}\left(B_{i}\right), s_{2}\left(B_{i}\right), \ldots\right.$, $\left.s_{t}\left(B_{i}\right)\right)$ of index $B_{i}$ in the second level, where $s_{k}\left(B_{i}\right)$ indicates the degree to which $B_{i}$ belongs to evaluation grade $\xi_{k}$. 
The grade vector $s(A)=\left(s_{1}(A), s_{2}(A), \ldots, s_{t}(A)\right)$ of the overall goal $A$ can be obtained in the same way.

Remark 25. From the property of $H_{i j}(A)$, it is obtained that the larger the $\lambda_{i j}(A)$ is, the more important the role $C_{i j}$ plays in the evaluation of $A$ is. If $\lambda_{i j}(A)=1$, then the evaluation grade of $A$ can be determined only by index $C_{i j}$. If $\lambda_{i j}(A)=0$, then index $C_{i j}$ does not play any role in determining the grade of $A$.

Theorem 26. The weighted-average method is a special case of the above method.

Proof. For each row vector $\left(s_{j 1}, s_{j 2}, \ldots, s_{j 5}\right)$, if there is one component $s_{j k}=1$ for some $k$ and the rest four components are 0 , then $H_{i j}(A)=1, V_{i j}(A)=1$, and $\lambda_{i j}(A)=1 / n$. Thus, by formulas

$$
\begin{gathered}
N_{k}\left(B_{i}\right)=\sum_{j=1}^{m_{i}} w_{i j}\left(B_{i}\right) \cdot \lambda_{i j}(A) \cdot s_{i j k}, \\
s_{k}\left(B_{i}\right)=\frac{N_{k}\left(B_{i}\right)}{\sum_{k=1}^{t} N_{k}\left(B_{i}\right)},
\end{gathered}
$$

we have

$$
s_{k}(A)=\sum_{j=1}^{n} \lambda_{j} \cdot s_{j k}, \quad k=1,2, \ldots, t .
$$

That is to say, the weighted-average method is a special case of the above method.

3.5. Identification. Sometimes, the evaluation scales are comparable (e.g., Poor, Fair,..., Excellent scale; "Fair" is better than "Poor"), and a partial order " $>$ " can be defined according to the actual situation. If evaluation scale $C_{1}$ is better than $C_{2}$, we denote $C_{1}>C_{2}$; otherwise we denote $C_{1} \prec C_{2}$. Of course, whether $C_{1}$ is better than $C_{2}$ depends on the actual situation. To address the ordered division (e.g., Poor, Fair,..., Excellent), Cheng [26] proposed the confidence degree principle.

Confidence Degree Principle (Cheng [26]). Let “ $>$ " be a partial order, let $C_{1}, C_{2}, \ldots, C_{t}$ be an ordered division, and let $\lambda$ $(0.5<\lambda<1)$ be the confidence level. If $C_{1}>C_{2}>\cdots>C_{t}$,

$$
k_{0}=\min \left\{k: \sum_{i=1}^{k} \mu_{x}\left(C_{i}\right) \geq \lambda, 1 \leq k \leq t\right\} \text {, }
$$

and if $C_{1} \prec C_{2} \prec \cdots \prec C_{t}$,

$$
k_{0}=\max \left\{k: \sum_{i=1}^{k} \mu_{x}\left(C_{i}\right) \geq \lambda, 1 \leq k \leq t\right\} \text {, }
$$

and then $x$ belongs to $C_{k_{0}}$ with at least the confidence level $\lambda$.

\section{An Application in the Evaluation of Software Quality}

In this section, an application of the proposed method in evaluation of software quality is given.
4.1. Evaluation System and Some Data. A problem of evaluating software quality was discussed in Li [27]. Next, we apply the proposed method to this evaluation problem, and the evaluation system is shown as in Table 2 . Based on the evaluation criteria, the experts provided the scores of the bottom indices in Column 5 of Table 2. The evaluation grades are "Poor," "Fair," "Good," "Very good," and "Excellent." They are represented by uncertain sets $\xi_{1}, \xi_{2}, \ldots, \xi_{5}$ with membership functions $\mu_{1}, \mu_{2}, \ldots, \mu_{5}$, respectively, where membership functions

$$
\begin{aligned}
& \mu_{1}(x)= \begin{cases}\frac{1,}{(1-x)} & \text { if } 0 \leq x \leq 0.2, \\
0, & \text { if } 0.2<x \leq 1,\end{cases} \\
& \mu_{2}(x)= \begin{cases}\frac{(x-0.2)}{0.8}, & \text { if } 0.2 \leq x \leq 1, \\
2-x, & \text { if } 1<x \leq 2, \\
0, & \text { otherwise, }\end{cases} \\
& \mu_{3}(x)= \begin{cases}\frac{x-1,}{3-x,} \text { if } 1 \leq x \leq 2, \\
\text { if } 2<x \leq 3,\end{cases} \\
& \mu_{4}(x)= \begin{cases}\frac{x-2,}{(3.8-x)} & \text { otherwise } \\
0.8, & \text { if } 3<x \leq 3.8, \\
0, & \text { otherwise, }\end{cases} \\
& \mu_{5}(x)= \begin{cases}\frac{(x-3)}{0.8,} & \text { if } 3 \leq x \leq 3.8, \\
1, & \text { if } 3.8<x \leq 4, \\
0, & \text { otherwise }\end{cases}
\end{aligned}
$$

are given by experts according to their personal knowledge and actual situation of the evaluation. According to the scores given by experts (Column 5 in Table 2) and the above membership functions, the grade vectors (Column 4 in Table 2) can be obtained (see Section 4.3).

4.2. Weights of Indices. There are ten experts in the evaluation group, and there are five degrees of importance (namely, "most important," "more important," "important," "less important," and "unimportant"), which are represented by uncertain variables $\eta_{1}, \eta_{2}, \ldots, \eta_{5}$ with the same uncertainty distribution $Z(0.54,0.69,0.84)$. Taking indices $C_{11}, C_{12}, C_{13}$, $C_{14}$, for examples, the evaluation results from the experts are shown as in Table 3. Weight $w_{1 j}$ of index $C_{1 j}$ is an uncertain variable $\eta_{1 j}$. By $\omega_{1 j}=\left(r_{1} / r\right) \eta_{1}+\cdots+\left(r_{n} / r\right) \eta_{n}, j=1,2,3,4$, and Theorem 8 , we have $\eta_{1 j} \sim Z(0.54,0.69,0.84)$. The vector $w_{1}=\left(w_{11}, w_{12}, w_{13}, w_{14}\right)$ is obtained by the 99-table, and the values of $w_{1 j}$ are shown in Table 4 .

By Example 11 and Theorem 12, the expected value of $w_{1}=\left(w_{11}, w_{12}, w_{13}, w_{14}\right)$ is $(0.78,0.78,0.78,0.78)$. Normalizing the expected value, the weights of indices $C_{11}, C_{12}$, $C_{13}, C_{14}$ are $(0.25,0.25,0.25,0.25)$. The weights of the other indices can be obtained in the same way (the numbers in Column 3 of Table 2). 
TABLE 2: Evaluation system of software quality.

\begin{tabular}{|c|c|c|c|c|}
\hline The overall goal & Second-level indices and weights & Bottom indices and weights & Grade vectors & Scores \\
\hline \multirow{24}{*}{ Software quality $A$} & \multirow{4}{*}{ Install section $B_{1}(0.1)$} & Install difficulty $C_{11}(0.25)$ & $(0,0,0.50,0.50,0)$ & 2.50 \\
\hline & & Size after installation $C_{12}(0.25)$ & $(0,0,0,1.00,0)$ & 3.00 \\
\hline & & Memory occupation $C_{13}(0.25)$ & $(0,0,0.30,0.70,0)$ & 2.70 \\
\hline & & Shortcuts creation $C_{14}(0.25)$ & $(0,0,0,0.50,0.50)$ & 3.40 \\
\hline & \multirow{5}{*}{ General structure $B_{2}(0.15)$} & Complete module $C_{21}(0.2)$ & $(0,0,0.41,0.59,0)$ & 2.59 \\
\hline & & Appropriate grade $C_{22}(0.2)$ & $(0,0,0,0.50,0.50)$ & 3.40 \\
\hline & & Reasonable module partition $C_{23}(0.2)$ & $(0,0,1.00,0,0)$ & 2.00 \\
\hline & & Business integration level $C_{24}(0.2)$ & $(0,0,0,0,1.00)$ & 4.00 \\
\hline & & Data import and extraction function $C_{25}(0.2)$ & $(0,0,0.30,0.70,0)$ & 2.70 \\
\hline & \multirow{4}{*}{ Security mechanism $B_{3}(0.15)$} & Permissions allocation $C_{31}(0.25)$ & $(0,1.00,0,0,0)$ & 1.00 \\
\hline & & Operating $\log C_{32}(0.25)$ & $(0,0,0,1.00,0)$ & 3.00 \\
\hline & & Data backup and restoration $C_{33}(0.25)$ & $(0,0,0.30,0.70,0)$ & 2.70 \\
\hline & & Data maintenance $C_{34}(0.25)$ & $(0,0,0.50,0.50,0)$ & 2.50 \\
\hline & \multirow{4}{*}{ Program and data processing $B_{4}(0.2)$} & Reliability $C_{41}(0.25)$ & $(0,0,0.30,0.70,0)$ & 2.70 \\
\hline & & Efficiency $C_{42}(0.25)$ & $(0,0,1.00,0,0)$ & 2.00 \\
\hline & & Maintainability $C_{43}(0.25)$ & $(0,0,1.00,0,0)$ & 2.00 \\
\hline & & Portability $C_{44}(0.25)$ & $(0,0,0,0,1.00)$ & 4.00 \\
\hline & \multirow{3}{*}{ Code maintenance $B_{5}(0.1)$} & Definable code $C_{51}(0.33)$ & $(0,0,0,1.00,0)$ & 3.00 \\
\hline & & Gradable code $C_{52}(0.33)$ & $(0,0,0.30,0.70,0)$ & 2.70 \\
\hline & & Proper code maintenance $C_{53}(0.34)$ & $(0,0,0.50,0.50,0)$ & 2.50 \\
\hline & \multirow{4}{*}{ Operating performance $B_{6}(0.3)$} & Friendly interface $C_{61}(0.25)$ & $(0,0,0.30,0.70,0)$ & 2.70 \\
\hline & & Easy-to-use $C_{62}(0.25)$ & $(0,0,0.50,0.50,0)$ & 2.50 \\
\hline & & Online help $C_{63}(0.25)$ & $(0,0,0.30,0.70,0)$ & 2.70 \\
\hline & & Multitasking switching $C_{64}(0.25)$ & $(0,0,1.00,0,0)$ & 2.00 \\
\hline
\end{tabular}

TABLE 3: Evaluation results given by experts.

\begin{tabular}{|c|c|c|c|c|c|}
\hline Bottom indices & Most important & More important & Important & Less important & Unimportant \\
\hline Install difficulty $C_{11}$ & 1 & 2 & 3 & 2 & 2 \\
\hline Size after installation $C_{12}$ & 2 & 1 & 3 & 2 & 2 \\
\hline Memory occupation $C_{13}$ & 2 & 2 & 3 & 1 & 2 \\
\hline Shortcuts creation $C_{14}$ & 2 & 3 & 2 & 2 & 1 \\
\hline
\end{tabular}

TABLE 4: The 99-table of weights for indices $C_{11}, C_{12}, C_{13}, C_{14}$.

\begin{tabular}{lllll}
\hline Weights & 0.01 & 0.02 & $\cdots$ & 0.99 \\
\hline$w_{11}$ & 0.54 & 0.55 & $\cdots$ & 0.84 \\
$w_{12}$ & 0.54 & 0.55 & $\cdots$ & 0.84 \\
$w_{13}$ & 0.54 & 0.55 & $\cdots$ & 0.84 \\
$w_{14}$ & 0.54 & 0.55 & $\cdots$ & 0.84 \\
\hline
\end{tabular}

4.3. Grade Vectors of Bottom Indices. Taking the grade vector of index $C_{11}$ as an example, the score value $x_{11}$ of index $C_{11}$ is 2.50 and the membership functions of evaluation grades $\xi_{1}, \xi_{2}, \ldots, \xi_{5}$ are $\mu_{1}, \mu_{2}, \ldots, \mu_{5}$, respectively. Therefore, the grade vector of index $C_{11}$ is

$$
\begin{aligned}
s\left(C_{11}\right) & =\left(\mu_{1}\left(x_{11}\right), \mu_{2}\left(x_{11}\right), \ldots, \mu_{5}\left(x_{11}\right)\right) \\
& =(0,0,0.50,0.50,0) .
\end{aligned}
$$

Similarly, the other grade vectors of the bottom indices can be obtained by the scores of the bottom indices (Column 5 in Table 2).

4.4. Grade Vector of Software Quality A. In this subsection, the transformation algorithm established above is used to realize the transformation from the grade vectors of the bottom indices to the grade vector of software quality.

4.4.1. Grade Vectors of Indices in the Second Level. We take the calculation of grade vector $s\left(B_{1}\right)$ of index $B_{1}$ (install section) as an example.

There are four indices, $C_{11}, C_{12}, C_{13}, C_{14}$, immediately below $B_{1}$, and the grade-transition matrix of $B_{1}$ is

$$
U\left(B_{1}\right)=\left(\begin{array}{ccccc}
0 & 0 & 0.50 & 0.50 & 0 \\
0 & 0 & 0 & 1.00 & 0 \\
0 & 0 & 0.30 & 0.70 & 0 \\
0 & 0 & 0 & 0.50 & 0.50
\end{array}\right) .
$$


TABLE 5: Comparison of the results with the other two methods.

\begin{tabular}{lccccc}
\hline Grades & Poor & Fair & Good & Very good & Excellent \\
\hline The proposed method & 0 & 0.0356 & 0.4319 & 0.4903 & 0.0422 \\
Fuzzy weighted-average method & 0 & 0.1051 & 0.4507 & 0.4053 & 0.0389 \\
Fuzzy max-min method & 0 & 0.0822 & 0.4165 & 0.4697 & 0.0516 \\
\hline
\end{tabular}

TABLE 6: The differences between two adjacent grades.

\begin{tabular}{|c|c|c|c|c|}
\hline Differences & $\xi_{1}$ and $\xi_{2}$ & $\xi_{2}$ and $\xi_{3}$ & $\xi_{3}$ and $\xi_{4}$ & $\xi_{4}$ and $\xi_{5}$ \\
\hline The proposed method & 0.0356 & 0.3963 & 0.0584 & 0.4481 \\
\hline Fuzzy weighted-average method & 0.1051 & 0.3456 & 0.0454 & 0.3664 \\
\hline Fuzzy max-min method & 0.0822 & 0.3343 & 0.0532 & 0.4181 \\
\hline
\end{tabular}

With the weights $\left(w_{11}, w_{12}, w_{13}, w_{14}\right)=(0.25,0.25,0.25$, 0.25 ) of indices $C_{11}, C_{12}, C_{13}, C_{14}$, we can obtain

$$
s\left(B_{1}\right)=(0,0,0.1706,0.7262,0.1032) .
$$

Similarly, we can obtain the grade vectors

$$
\begin{gathered}
s\left(B_{2}\right)=(0,0,0.3777,0.2815,0.3408), \ldots, \\
s\left(B_{6}\right)=(0,0,0.5896,0.4104,0) .
\end{gathered}
$$

4.4.2. Grade Vector of Software Quality $A$. With the grade vectors $s\left(B_{1}\right), \ldots, s\left(B_{6}\right)$ of indices $B_{1}, \ldots, B_{6}$, we can obtain the grade-transition matrix of $A$ as

$$
U(A)=\left(\begin{array}{ccccc}
0 & 0 & 0.1706 & 0.7262 & 0.1032 \\
0 & 0 & 0.3777 & 0.2815 & 0.3408 \\
0 & 0.3135 & 0.1476 & 0.5389 & 0 \\
0 & 0 & 0.6038 & 0.3962 & 0 \\
0 & 0 & 0.2172 & 0.7828 & 0 \\
0 & 0 & 0.5896 & 0.4104 & 0
\end{array}\right)
$$

Similar to calculation (1), the grade vector $s(A)$ of the overall goal is obtained as

$$
s(A)=(0,0.0356,0.4319,0.4903,0.0422) .
$$

4.5. Identification. In this example, the confidence level of $A$ belonging to $\xi_{4}$ (Very good) is no less than $0.53(0.4903+$ $0.0422 \geq 0.53)$ and the confidence level of $A$ belonging to $\xi_{3}$ (Good) is no less than $0.96(0.4903+0.0422+0.4319 \geq 0.96)$. Comparatively, it is more reasonable to define $A$ in grade $\xi_{4}$. Therefore, the quality of this software is "Very good."

4.6. Comparative Analysis. In $\mathrm{Li}$ [27], the fuzzy max-min method is used in the software quality evaluation, and we calculate the results via the fuzzy weighted-average method with the same data in Table 2. The results are shown in Table 5. Table 5 shows that the proposed method and fuzzy maxmin method produced the same result (i.e., the evaluation result is Very good) and the evaluation result from fuzzy weighted method is Good. And also there is some difference in the membership degree of each grade. Furthermore, the literatures $[28,29]$ discussed the algorithms for maximizing the soft margin, which show that the larger the difference between two adjacent grades is, the stronger the classification ability of the method is. Therefore, we analyzed the differences between two adjacent grades in Table 6. From Table 6, we can see that there are three differences (between $\xi_{4}$ and $\xi_{5}$, $\xi_{3}$ and $\xi_{4}$, and $\xi_{2}$ and $\xi_{3}$ ) and the proposed method is larger than the other two methods. Thus, the proposed method has a stronger classification ability in the evaluation of software quality.

\section{Conclusions}

In this paper, weights for bottom indices with respect to the overall goal in an evaluation system are introduced and a new uncertainty evaluation method is proposed based on uncertain sets and uncertain variables. This method generalizes the weighted average method, and it is applied in the evaluation of software quality. Comparative analysis with other two methods shows that the proposed method has a stronger classification ability in the evaluation of software quality. More importantly, the proposed method in this paper can also be used in other evaluation systems with a hierarchical structure. Therefore, more applications of the proposed method can be underdeveloped further.

\section{Conflict of Interests}

The authors declare that there is no conflict of interests regarding the publication of this paper.

\section{Acknowledgments}

This work is supported by the National Natural Science Foundation of China (no. 61073121), the Natural Science Foundation of Hebei Province of China (nos. F2012402037, A2012201033), and the Natural Science Foundation of Hebei Education Department (no. Q2012046). The authors also thank anonymous reviewers for their constructive comments and suggestions and acknowledge the English language editing by Elseviers WebShop. 


\section{References}

[1] T. Saaty, "Modeling unstructured decision problems-the theory of analytical hierarchies," Mathematics and Computers in Simulation, vol. 20, no. 3, pp. 147-158, 1978.

[2] H. H. Wu and Y. N. Tsai, "An integrated approach of AHP and DEMATEL methods in evaluating the criteria of auto spare parts industry," International Journal of Systems Science, vol. 43, no. 11, pp. 2114-2124, 2012.

[3] M. Kumar, S. P. Yadav, and S. Kumar, "Fuzzy system reliability evaluation using time-dependent intuitionistic fuzzy set," International Journal of Systems Science, vol. 44, no. 1, pp. 50-66, 2013.

[4] X. Zhao and L. Cui, "Reliability evaluation of generalised multistate k-out-of-n systems based on FMCI approach," International Journal of Systems Science, vol. 41, no. 12, pp. 1437-1443, 2010.

[5] D. Chen, M. Fu, X. Jiang, and D. Song, “The investment decision-making index system and the grey comprehensive evaluation method under hybrid cloud," Advances in Computer Science and Information Engineering, vol. 169, no. 2, pp. 171-176, 2012.

[6] C. Lee, H. Lee, H. Seol, and Y. Park, "Evaluation of new service concepts using rough set theory and group analytic hierarchy process," Expert Systems with Applications, vol. 39, no. 3, pp. 3404-3412, 2012.

[7] X. Geng, X. Chu, and Z. Zhang, "A new integrated design concept evaluation approach based on vague sets," Expert Systems with Applications, vol. 37, no. 9, pp. 6629-6638, 2010.

[8] M. Mareš, "Information measures and uncertainty of particular symbols," Kybernetika, vol. 47, no. 1, pp. 144-163, 2011.

[9] I. Kramosil, "Decision-making under uncertainty processed by lattice-valued possibilistic measures," Kybernetika, vol. 42, no. 6, pp. 629-646, 2006.

[10] H. Y. Li, Y. A. Hu, and R. Q. Wang, "Adaptive finite-time synchronization of cross-strict feedback hyperchaotic systems with parameter uncertainties," Kybernetika, vol. 49, no. 4, pp. 554567, 2013.

[11] Y. Lan, R. Zhao, and W. Tang, "A bilevel fuzzy principal-agent model for optimal nonlinear taxation problems," Fuzzy Optimization and Decision Making, vol. 10, no. 3, pp. 211-232, 2011.

[12] L. A. Zadeh, "Fuzzy sets," Information and Computation, vol. 8, pp. 338-353, 1965.

[13] B. D. Liu, Uncertainty Theory, Springer, Berlin, Germany, 2007.

[14] B. D. Liu, Uncertainty Theory: A Branch of Mathematics for Modeling Human Uncertainty, Springer, Berlin, Germany, 2010.

[15] D. Kahneman and A. Tversky, "Prospect theory: an a nalysis of decision under risk," Econometrica, vol. 47, no. 2, pp. 263-292, 1979.

[16] B. D. Liu, Theory and Practice of Uncertain Programming, Springer, Berlin, Germany, 2009.

[17] B. D. Liu, "Uncertain risk analysis and uncertain reliability analysis," Journal of Uncertain Systems, vol. 4, pp. 163-170, 2010.

[18] B. D. Liu, "Uncertain logic for modeling human language," Journal of Uncertain Systems, vol. 5, no. 1, pp. 3-20, 2011.

[19] B. D. Liu, "Uncertain set theory and uncertain inference rule with application to uncertain control," Journal of Uncertain Systems, vol. 4, pp. 83-98, 2010.

[20] B. D. Liu, "Extreme value theorems of uncertain process with application to insurance risk model," Soft Computing, vol. 17, no. 4, pp. 549-556, 2013.
[21] B. D. Liu, Uncertainty Theory, 4th edition, 2013, http://orsc.edu .cn/liu/ut.pdf.

[22] J. J. Liu, "Uncertain comprehensive evaluation method," Journal of Information and Computational Science, vol. 8, no. 2, pp. 336344, 2011.

[23] B. D. Liu, "Some research problems in uncertainty theory," Journal of Uncertain Systems, vol. 3, pp. 3-10, 2009.

[24] B. D. Liu, "Membership functions and operational law of uncertain sets," Fuzzy Optimization and Decision Making, vol. 11, no. 4, pp. 387-410, 2012.

[25] B. D. Liu, "A new definition of independence of uncertain sets," Fuzzy Optimization and Decision Making, vol. 12, no. 4, pp. 451461, 2013.

[26] Q. S. Cheng, "A model of property identification and its application," Acta Scientiarum Naturalium Universitatis Pekinensis, vol. 33, pp. 12-20, 1997.

[27] L. Li and X. Han, "Multilevel fuzzy integrated evaluation of software quality," Journal of Harbin Institute of Technology, vol. 35, no. 7, pp. 812-819, 2003.

[28] N. Cristianini and J. S. Taylor, An Introduction to Support Vector Machines and Other Kernel-Based Learning Methods, Cambridge University Press, New York, NY, USA, 2000.

[29] J. Shawe-Taylor and N. Cristianini, "On the generalization of soft margin algorithms," IEEE Transactions on Information Theory, vol. 48, no. 10, pp. 2721-2735, 2002. 


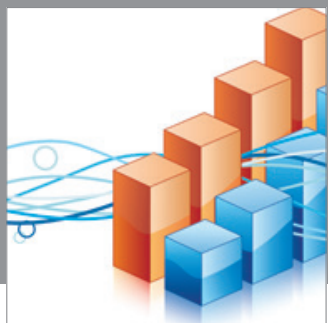

Advances in

Operations Research

mansans

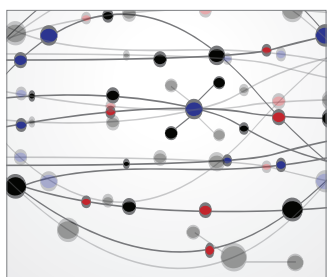

The Scientific World Journal
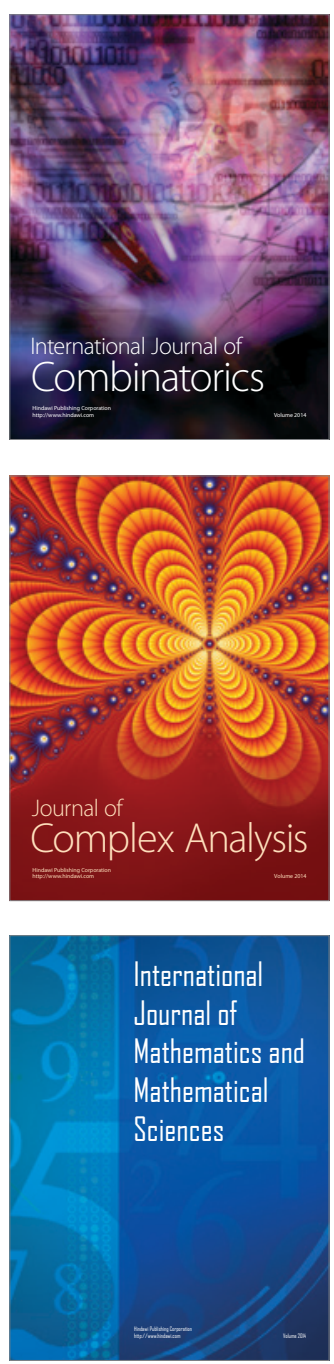
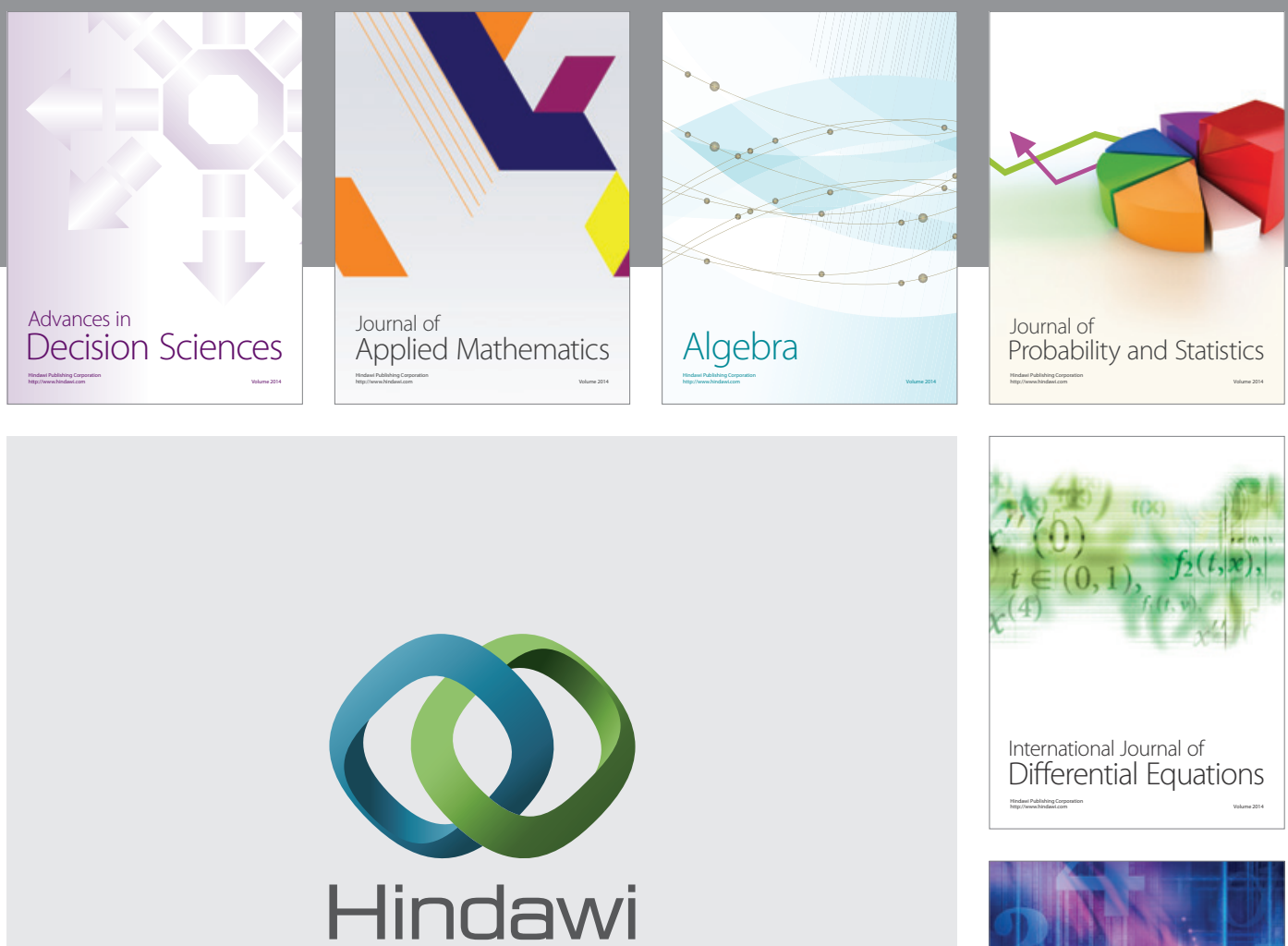

Submit your manuscripts at http://www.hindawi.com
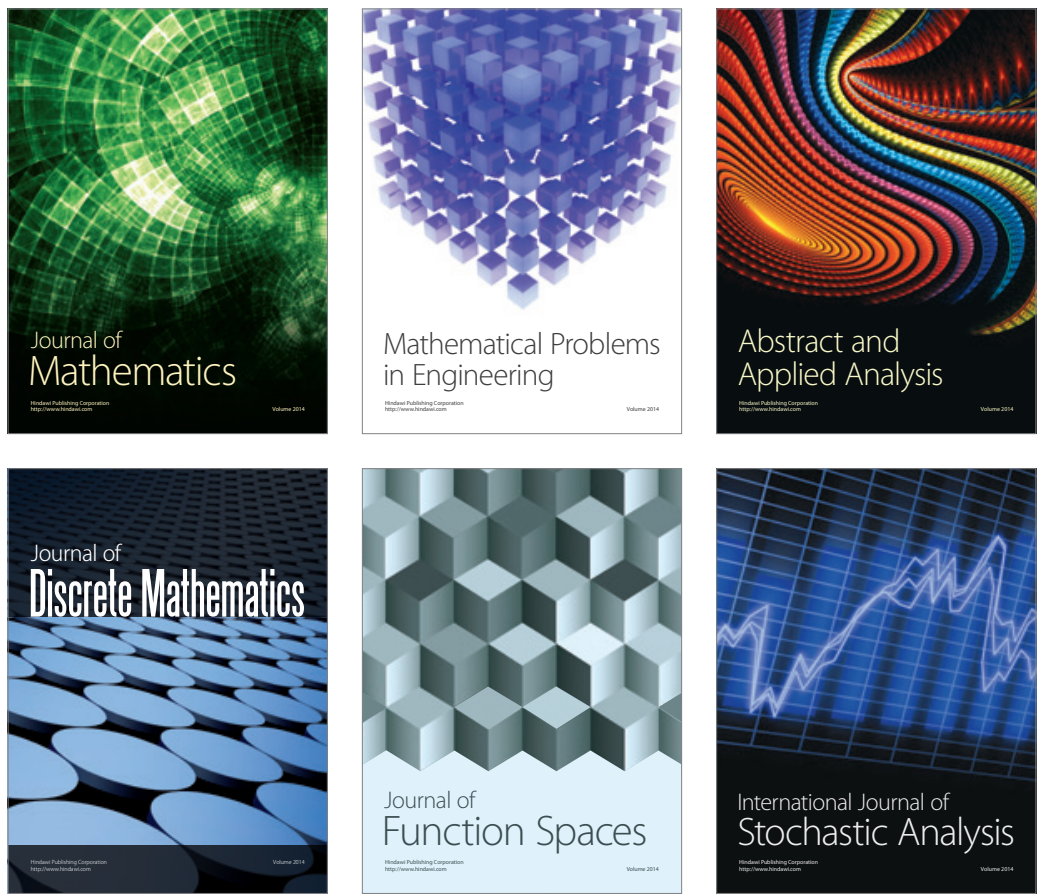

Journal of

Function Spaces

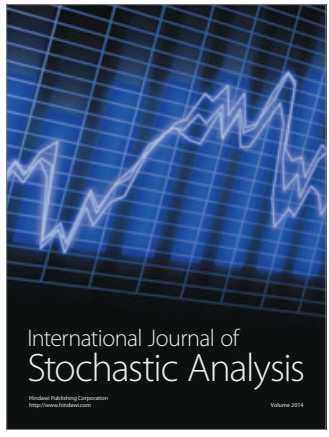

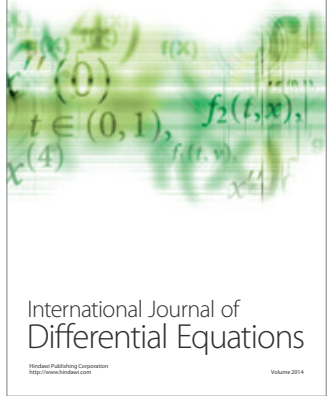
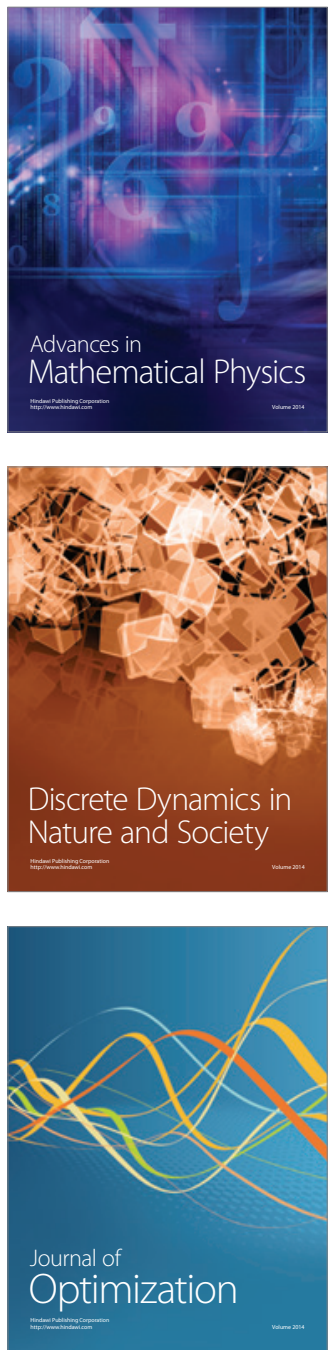